\title{
Modelo ontológico para representar información sobre la práctica profesional en una institución educativa
}

\author{
Juan Carlos Flores, Mireya Tovar, Ana-Patricia Cervantes \\ Benémerita Universidad Autónoma de Puebla, \\ Facultad de Ciencias de la Computación, Puebla, Mexico \\ \{fmjuancarlos\}@gmail.com, \{mtovar, patty\}@cs.buap.mx
}

\begin{abstract}
Resumen. En este artículo se presenta el diseño de un modelo ontológico para la búsqueda de información dentro de una institución educativa de nivel superior. Este modelo pretende brindar respuestas sobre los procesos de inscripción y liberación de las prácticas profesionales, es decir, de los requisitos que se deben llevar a cabo por el alumno para realizar este tipo de procedimientos en la institución educativa. En este trabajo se siguen las fases de diseño de la metodología propuesta por Grüninger y Fox's para la creación manual de la ontología; también se incluye la propuesta de un escenario, preguntas de competencia, definición de clases, propiedades, formalización y evaluación a través de las respuestas obtenidas por medio del lenguaje de consulta SPARQL.
\end{abstract}

Palabras clave: Metodologías, institución educativa, ontología.

\section{Ontological Model to Represent Information about the Professional Practice in an Educational Institution}

\begin{abstract}
This article presents the design of an ontological model for the search of information within a higher education institution. This model aims to provide answers about the processes of enrollment and release of professional practices, that is, the requirements that must be carried out by the student to perform this type of procedures in the educational institution. In this work the design phases of the methodology proposed by Grninger and Fox's for the manual creation of the ontology are followed; also includes the proposal of a scenario, questions of competence, the definition of classes, properties, formalization and evaluation through the answers obtained through the SPARQL query language.
\end{abstract}

Keywords. Methodologies, educational institution, ontology. 


\section{Introducción}

Hoy en día las universidades son un sector que genera una gran cantidad de datos o información proveniente de distintas áreas de trabajo, dicha información en la mayoría de los casos no es gestionada de una forma adecuada y mucho menos es accesible para todo público que requiere de ella [6]. Hoy en día existen fuentes de información en la Web que poseen motores de búsqueda que dan respuesta a lo que solicita el usuario, sin embargo, estos motores funcionan sintácticamente, es decir, devuelven resultados que contienen elementos léxicos relacionados exactamente con los términos de la consulta, es por ello que se recurre a la Web Semántica la cual propone superar las limitaciones de la Web actual. Dados estos cambios es imprescindible unificar el contenido a través de un lenguaje común y para ello se utiliza la noción de ontología del campo de la inteligencia artificial. Gruber define una ontología como "a formal explicit specification of a shared conceptualization" [5]. Una ontología define una jerarquía de conceptos, relaciones, restricciones, axiomas e instancias para describir un dominio, el cual servirá para el intercambio de la información. Por otra parte el uso de las ontologías hoy en día es muy común en áreas de negocios, finanzas, internet, medicina e industria como integradoras de fuentes de datos, así como de organización y representación de conocimiento. Para este trabajo se presenta el diseño de un modelo ontológico implementado en la herramienta Protégé [7]. La finalidad es que usuarios que necesiten realizar el proceso de práctica profesional puedan consultar toda la información relacionada con dicho proceso, que consta de una serie de pasos los cuales pueden ser representados en una ontología. El documento se encuentra estructurado de la siguiente manera: En la Sección 2. se presentan algunos trabajos relacionados con la creación manual de ontológias. En la Sección 3. se presenta el planteamiento del escenario y las preguntas de competencia. En la Sección 4. se presenta el diseño de la ontología y en la Sección 5. se muestran los resultados de la evaluación. Finalmente las conclusiones se presentan en la Sección 6. .

\section{Trabajos relacionados}

En esta sección se mencionan algunos trabajos que estan relacionados con el tema de búsqueda de información dentro de una institución educativa y creación manual de ontologías.

En Báez Bagatella, Tamborrell Hernández, Lasserre Chávez, Ramos Flores, Tovar Vidal, y Vilariño Ayala [1] se propone una ontología que contiene información relacionada con profesores, alumnos, personal administrativo, planes de estudio, infraestructura, proyectos y líneas de investigación de una institución educativa superior del estado de Puebla; se pretende que dicha información se encuentre de forma estructurada y que pueda ser consultada por humanos y otros sistemas computacionales.

El modelo ontológico propuesto se divide en planteamiento, descripción de las entidades y clases de equivalencia. En Rose Gómez, Serna Encinas y Rodríguez 
Elías [9] se presenta el uso del lenguaje natural para la búsqueda de información de una organización académica, para la creación del modelo de conocimiento. Se utiliza la metodología KoFI que se compone de cuatro etapas: Identificación de fuentes de conocimiento, Identificación de tipos/temas de conocimiento, identificación de flujos de conocimiento e identificación de problemas que afecten el flujo de conocimiento. Con el análisis de las cuatro etapas se obtiene un meta modelo que es la base del modelo de conocimiento, el cual considera un repositorio de datos y un repositorio de conocimiento.

El repositorio de datos incluye una base de datos para los documentos y para el repositorio de conocimiento se usa una ontología diseñada, a través de la metodología Methontology e implementada con la herramienta Protégé. En Rosell León, Senso Ruiz y Leiva Maderos [10] se describe el desarrollo de una ontología nombrada UH-Ontology para el manejo de datos de una universidad. La metodología que se utiliza para la elaboración de UH-Ontology consta de siete pasos; 1) Determinar el dominio y el alcance de la ontología, 2) Reutilizar ontologías ya existentes, 3) Enumerar términos importantes, 4) Definir clases y jerarquía de clases, 5) Definir propiedades de clases (SLOTS), 6) Definir facetas de los slots y 7) Crear instancias.

La herramienta utilizada para la modelación e implementación de la ontología fue Protégé 4.3. En Tabares García, y Jiménez Builes [11] se presenta la construcción de una ontología para el proceso evaluativo en la educación superior, para este trabajo se utilizá la metodología de Methontology y la herramienta de Protégé 3.4.8. En Mora Arciniega y Segarra Faggioni [6] se describe la realización de un modelo ontológico para la representación de datos académicos y su publicación con tecnología semántica utilizando los conceptos de ontologías y datos enlazados, la propuesta contempla el desarrollo de una ontología para representar los datos de los planes de un curso y la publicación de estos datos mediante las mejores prácticas de Datos Enlazados. En Bravo, Martínez Reyes y Rodríguez [2] se describe un modelo ontológico para la representación de contexto académico e institucional. El sistema ofrece un contenido base que provee respuestas a las cuestiones académicas como tutorías, supervisión de tesis, además de lo relativo a ubicación de personas, bibliotecas, edificios, carreteras, horarios de clase y horarios de eventos.

El principal objetivo del modelo ontológico es mostrar las ventajas soluciones basadas en ontologías, dicho modelo esta implementado en el idioma español, el modelo ontológico integra tres ontologías las cuales son AreaGeografica, Persona y Academica. De igual manera presentan la evaluación de la ontología por medio de convertir preguntas de competencia en consultas SQRWL, dichas consultas pueden ser ingresadas en el sistema para dar respuesta a las preguntas. El modelo ontológico es implementado a través de una interfaz móvil e interfaces de escritorio donde el usuario puede interactuar con el modelo, se muestra un ejemplo donde un estudiante puede obtener la oficina y los salones de clases de un profesor. En Tovar, Flores y Reyes-Ortiz [12] se presenta un método para construir una ontología manual para la búsqueda de información sobre los trámites que debe realizar los estudiantes de una institución educativa para registrar y liberar 
su servicio social. Se presentan los pasos a seguir de la metodología Grüninger y Fox's [4] y Bravo et. al., [2] para la creación manual de la ontología. En Reyes, Tovar y Vázquez [8] se presenta un método para construir una ontología manual para un programa de maestría en Ciencias de la Computación utilizando las metodologías de METHONTOLOGY, Grüninger y Fox's [4] y Bravo et. al., [2].

En este trabajo de investigación se presenta el diseño ontológico de las activides que debe realizar un estudiante de nivel superior para registrar y liberar su práctica profesional en una institución educativa. El modelo ontológico sigue los pasos de diseño de las metodologías de Grüninger y Fox's [4] y Bravo et. al., $[2]$.

\section{Propuesta de modelo ontológico}

La construcción de sistemas basados en ontologías requiere una metodología clara, puesto que no solamente se trata de la construcción de un sistema, sino también de la generación de un modelo de experimentación e investigación. Se propone la metodología de Grüninger y Fox's y Bravo et. al.[2], para la realización de este trabajo, como ya se mencionó anteriormente. La metodología de Grüninger y Fox's se desarrolla en la universidad de Toronto y está inspirada para el desarrollo de conocimiento basado en sistemas usando lógica de primer orden.

La metodología propone identificar los principales escenarios como primer paso, es decir, posibles aplicaciones en las cuales se utilizará la ontología. Como segundo paso de la metodología se propone realizar una serie de preguntas denominadas como de "competencia", las cuales serán utilizadas para determinar el alcance de la ontología, estas preguntas y sus repuestas son usadas para extraer los principales conceptos y sus propiedades, relaciones y axiomas formales de la ontología [4].

\subsection{Planteamiento del escenario}

El modelo ontológico brindará respuesta acerca de los trámites y requisitos que se necesitan para que un alumno de una institución educativa de nivel superior pueda realizar sus prácticas profesionales. En el caso de los requisitos el alumno podrá visualizar la lista de los documentos necesarios para cada uno de los procesos que conllevan las prácticas profesionales, los cuales son el proceso de inscripción y el proceso de liberación. De igual manera se pondrá a su alcance un listado de programas los cuales son ofertas ofrecidas por los sectores empresarial y educativo para que el alumno realice sus prácticas profesionales con ellos. En dichas ofertas se coloca todo la información relacionada como por ejemplo: nombre del programa, número de solicitantes, ubicación del lugar donde se realizarán las prácticas, nombre de la persona encargada, etc. 
Modelo ontológico para representar información sobre la práctica profesional ...

\subsection{Preguntas de competencia}

Para la realización de este modelo ontológico y siguiendo con la metodología se elaboraron una serie de preguntas denomindas "preguntas de competencia", dichas preguntas se elaboran con la finalidad de tener una idea de lo que un usuario podría realizar como búsqueda dentro del modelo ontológico, además de que nos ayudarán a identificar clases, relaciones, propiedades y axiomas para la realización de la ontología. Entre las preguntas que se elaboraron se tienen las siguientes:

1. ¿Cuál es la oficina del coordinador de práctica profesional?

2. ¿Cuántas horas son en total de práctica profesional?

3. ¿Qué documentación necesito para inscribir la práctica profesional?

4. ¿Qué documentación necesito para liberar la práctica profesional?

5. ¿Cuántos créditos tiene la práctica profesional?

6. ¿En qué periodo puedo inscribir la práctica profesional?

7. ¿Cuál es el horario de atención del coordinador de práctica profesional?

8. ¿Cuáles son los tipos de programas que existen en la práctica profesional?

\subsection{Descripción de clases}

En esta fase se realizó un análisis, partiendo del escenario y de las preguntas de competencia, se identificaron las clases que contendría el modelo ontológico, de igual manera se obtuvieron las relaciones y las propiedades de cada clase. En la Tabla ?? se describen las clases identificadas incluidas en el modelo ontológico y su descripción.

En las Tablas 1 y 2 se describen las propiedades de cada una de las clases, las cuales ayudaron a almacenar información para dar respuesta a las preguntas de competencia. En la Tabla 1 se muestran las propiedades de la clase "Persona", así como de sus subclases. En la tabla 2 se muestran las propiedades de las clases restantes que son "PracticaProfesional, Programa, Facultad, Tramite y Requisito", así como de sus subclases.

\section{Diseño}

En este apartado se presenta el diseño de la ontología propuesta, el diseño se realizó con la herramienta Protégé, la cual es un software de código abierto que permite construir una ontología mediante su interfaz que ayuda al desarrollador en el proceso [7]. En la Fig. 1 se presentan las clases con las que cuenta el modelo ontológico. Las clases tienen relaciones entre ellas, es por ello que en la Fig. 2 se muestra el diagrama de las relaciones o propiedades de objeto y se describen en la Tabla 3 . 
Tabla 1. Propiedades de tipo de dato de la clase Persona.

\begin{tabular}{llr}
\hline $\begin{array}{l}\text { Propiedad de tipo Dominio } \\
\text { de Dato }\end{array}$ & & Rango \\
\hline \hline tieneNombre & Persona & String \\
tieneDireccion & Persona & String \\
tieneTelefono & Persona & String \\
tieneSexo & Persona & String \\
tieneEmail & Persona & String \\
tieneMatricula & Alumno & String \\
tieneHorarioAtencion & CoordinadorPracticaProfesional & String \\
tieneNoTrabajador & ResponsablePrograma, CoordinadorPracticaProfesional, String \\
& ProfesorResponsable & \\
tieneOficina & ResponsablePrograma, CoordinadorPracticaProfesional, String \\
& ProfesorResponsable & \\
\hline \hline
\end{tabular}

Tabla 2. Propiedades de tipo de dato de las clases PracticaProfesional, Programa, Facultad, Tramite y Requisito.

\begin{tabular}{lll}
\hline \hline Propiedad de Dato & Domino & Rango \\
\hline \hline tieneNombreFacultad & Facultad & String \\
tieneNombreCarrera & ProgramaDeEstudio & String \\
tieneNombrePrograma & Programa & String \\
tieneNoPrograma & Programa & String \\
tieneFechaRegistro & Programa & String \\
tieneEstatus & Programa & String \\
tieneFechaInicio & PracticaProfesional & String \\
tieneFechaTermino & PracticaProfesional & String \\
tieneCredito & PracticaProfesional & positiveInteger \\
tienePeriodo & PracticaProfesional & String \\
tieneDuracion & Programa & String \\
tieneDuracionPP & PracticaProfesional & String \\
tienePeriodoActual & Programa & String \\
tieneRequisitoInscripcion & Inscripcion & String \\
tieneRequisitoLiberacion & Liberacion & String \\
tieneTipo & Programa & String \\
\hline \hline
\end{tabular}




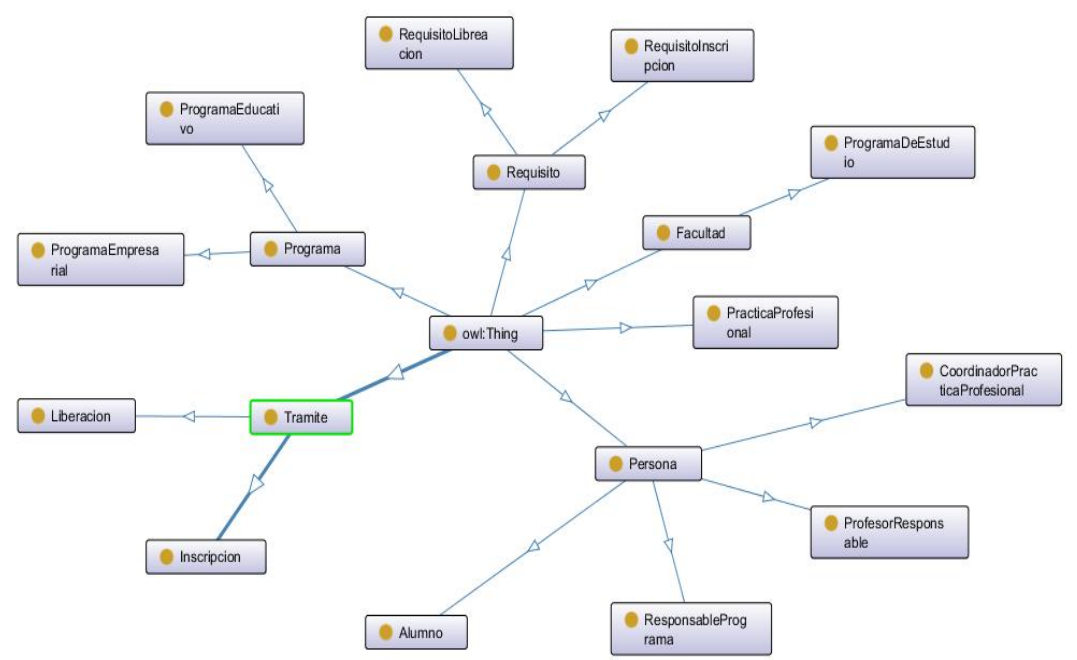

Fig. 1. Clases del modelo ontológico.

Tabla 3. Propiedades de objeto o relaciones entre clases.

\begin{tabular}{lll}
\hline \hline Object Properties & Domino & Rango \\
\hline \hline acargoDe & ProfesorResponsable & Alumno \\
autoriza & CoordinadotPracticaProfesional & $\begin{array}{l}\text { Liberacion, } \\
\text { Inscripcion }\end{array}$ \\
dirigidoA & Programa & ProgramaDeEstudio \\
inscritoEn & Alumno & ProgramaDeEstudio \\
realiza & Alumno & Inscripcin \\
registraUn & ResponsablePrograma & Programa \\
tiene & Liberacion, Inscripcion & RequisitoLiberacion, \\
& & RequisitoInscripcion \\
esRegistradoPor & Programa & ResponsablePrograma \\
\hline \hline
\end{tabular}




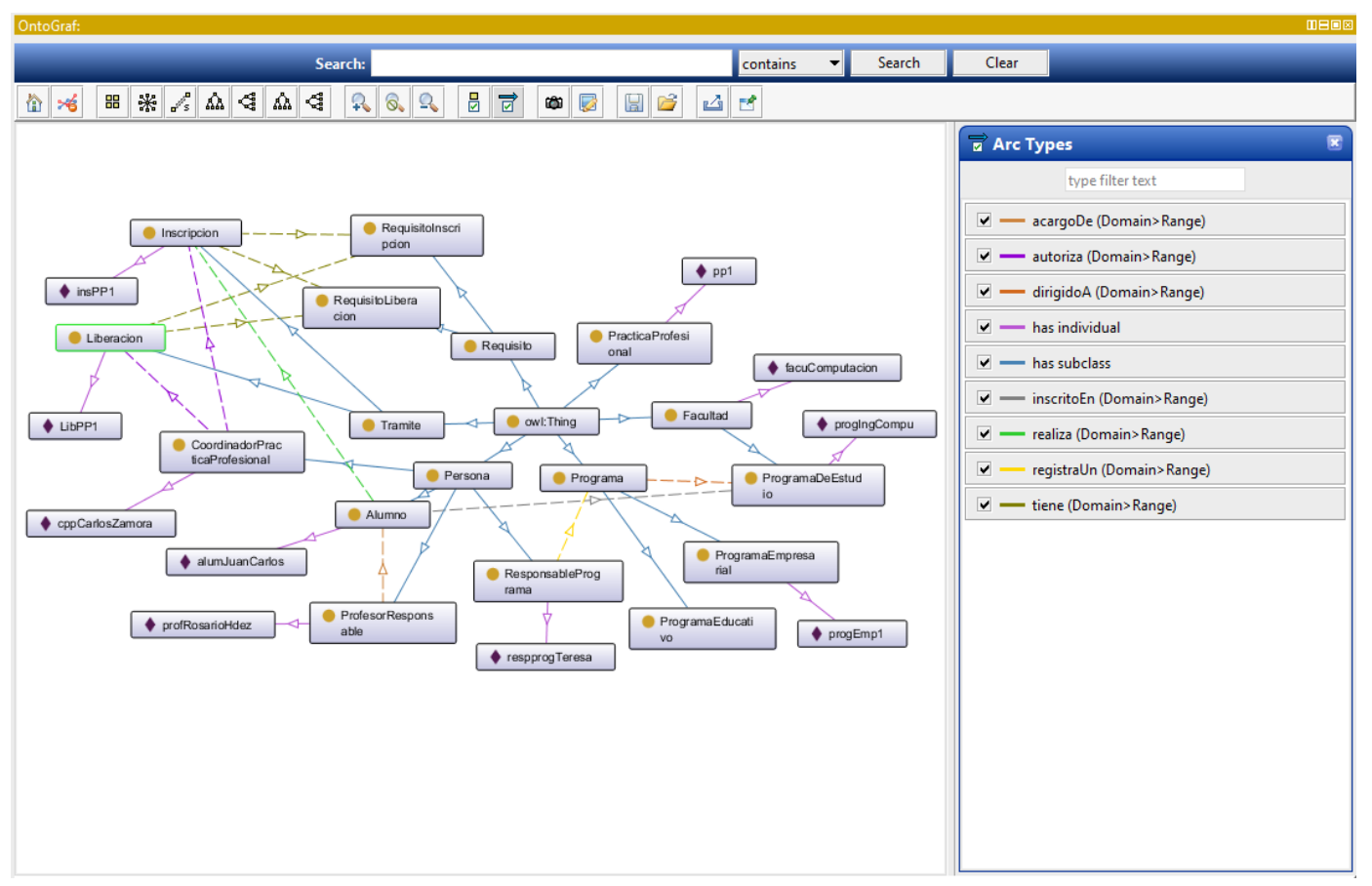

Fig. 2. Relaciones entre las clases del modelo ontológico.

\section{Resultados}

En esta sección se presenta la formalización de las preguntas de competencia (ver Tabla 4) las cuales se presentaron en la sección 3.2. La evaluación de la ontología se realizó a nivel de aplicación, es decir, a través de las respuestas que el sistema ontológico provee ante la formalización de las preguntas de competencia.

En este caso se utiliza el lenguaje de consulta SPARQL [3] y las respuestas de las preguntas de competencia son presentadas en las Fig. 3-10 (ver columna 3 de la Tabla 4 ).

\section{Conclusión}

La realización de esta ontología pretende que la información este organizada de forma más adecuada y que sea más fácil acceder a ella. Las ontologías son poderosas herramientas para estructurar la información, y muy utilizadas en ingeniería del conocimiento, procesamiento de lenguaje natural, inteligencia artificial, recuperación de información, análisis de sentimientos, etc. La principal contribución de este trabajo es la aplicación de una metodología para la creación manual de una ontología aplicada al dominio educativo para la búsqueda de 
Modelo ontológico para representar información sobre la práctica profesional ...

Tabla 4. Formalización de las preguntas de competencia y respuestas en SPARQL.

\begin{tabular}{|l|l|l|}
\hline \hline No & Pregunta y formalización & $\begin{array}{l}\text { Consulta } \\
\text { SPARQL }\end{array}$ \\
\hline \hline 1 & $\begin{array}{l}\text { ¿Cuál es la oficina del coordinador de práctica profesional? } \\
\exists \$ x \$ y \$ n \\
\text { tieneNombre }(\$ x, \$ n)) \wedge \text { tieneOficina }(\$ x, \$ y)) ?\end{array}$ & Fig. 3 \\
\hline 2 & $\begin{array}{l}\text { ¿Cuántas horas son en total de páctica profesional? } \\
\exists \$ x \$ y(\text { PracticaProfesional }(\$ x) \wedge \text { tieneDurationPP }(\$ x, \$ y)) ?\end{array}$ & Fig 4 \\
\hline 3 & $\begin{array}{l}\text { ¿Qué documentación necesito para inscribir la práctica profesional? } \\
\exists \$ x \$ y(\text { Inscripcion }(\$ x) \wedge \text { tieneRequisitoInscripcion }(\$ x, \$ y)) ?\end{array}$ & Fig. 5 \\
\hline 4 & $\begin{array}{l}\text { ¿Qué documentación necesito para liberar la práctica profesional? } \\
\exists \$ x \$ y(\text { Liberacion }(\$ x) \wedge \text { tieneRequisitoLiberacion }(\$ x, \$ y)) ?\end{array}$ & Fig. 6 \\
\hline 5 & $\begin{array}{l}\text { ¿Cuántos creditos tienen la práctica profesional? } \\
\exists \$ x \$ y(\text { PracticaProfesional }(\$ x) \wedge \text { tieneCredito }(\$ x, \$ y)) ?\end{array}$ & Fig. 7 \\
\hline 6 & $\begin{array}{l}\text { ¿En qué periodos puedo inscribir la práctica profesional? } \\
\exists \$ x \$ y(\text { PracticaProfesional }(\$ x) \wedge \text { tienePeriodo }(\$ x, \$ y)) ?\end{array}$ & Fig. 8 \\
\hline 7 & $\begin{array}{l}\text { ¿Cuál es horario de atención del coordinador de la práctica profesional? } \\
\exists \$ x \$ y \text { (CoordinadorPracticaProfesional }(\$ x) \\
\text { tieneNombre }(\$ x, \$ n)) \wedge \text { tieneHorarioAtencion }(\$ x, \$ y)) ?\end{array}$ & Fig. 9 \\
\hline 8 & $\begin{array}{l}\text { ¿Cuáles son los tipos de programas que existen en la práctica profe- } \\
\text { sional? } \\
\exists \$ x(\text { Programa }(\$ x)) ?\end{array}$ & Fig. 10 \\
\hline \hline
\end{tabular}

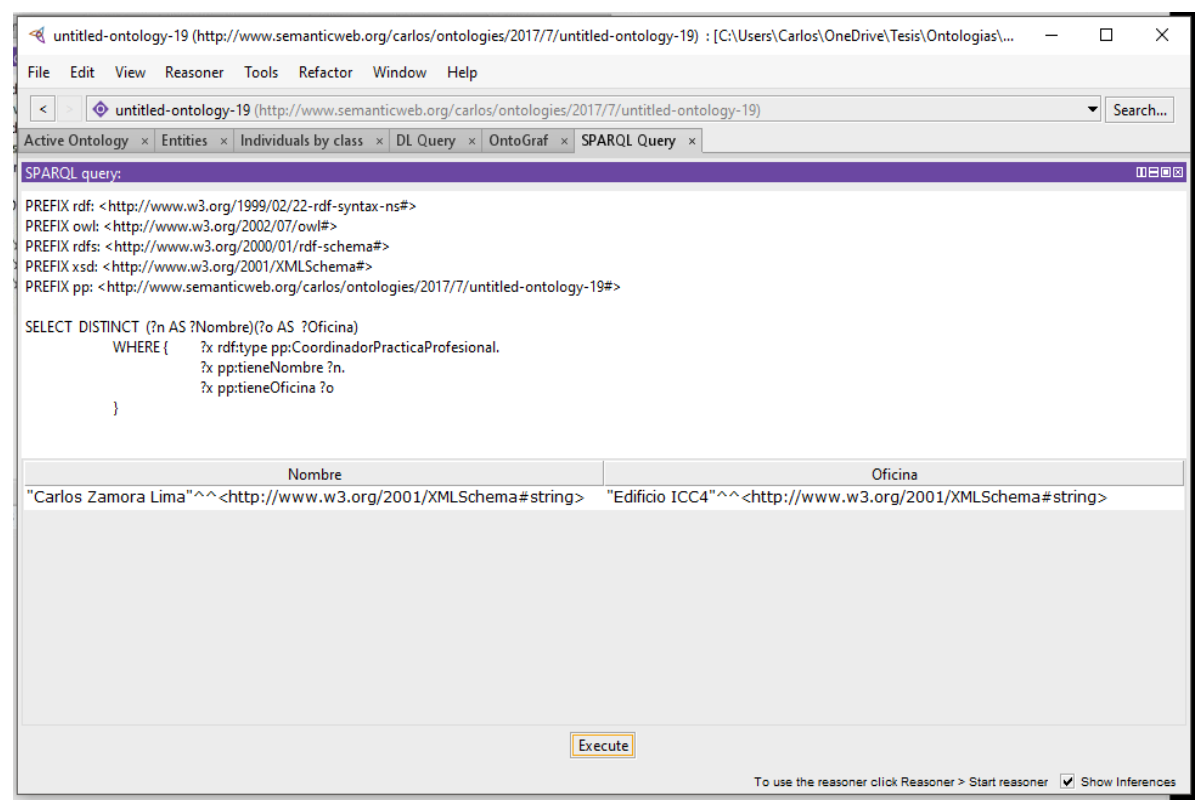

Fig. 3. Respuesta a la pregunta 1. 
Juan Carlos Flores Molina, Mireya Tovar Vidal, Ana Patricia Cervantes Márquez

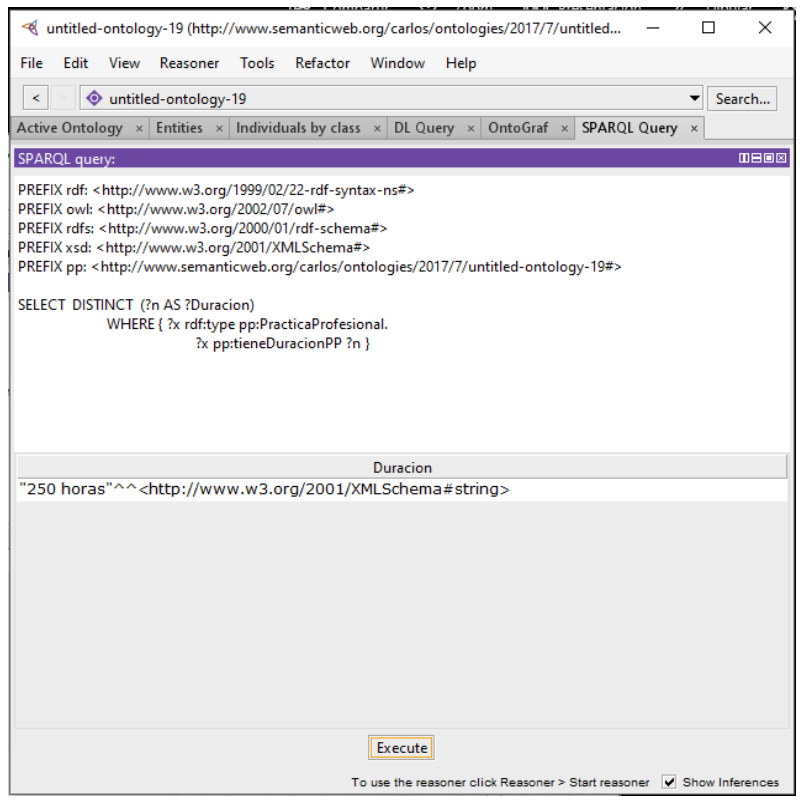

Fig. 4. Respuesta a la pregunta 2.

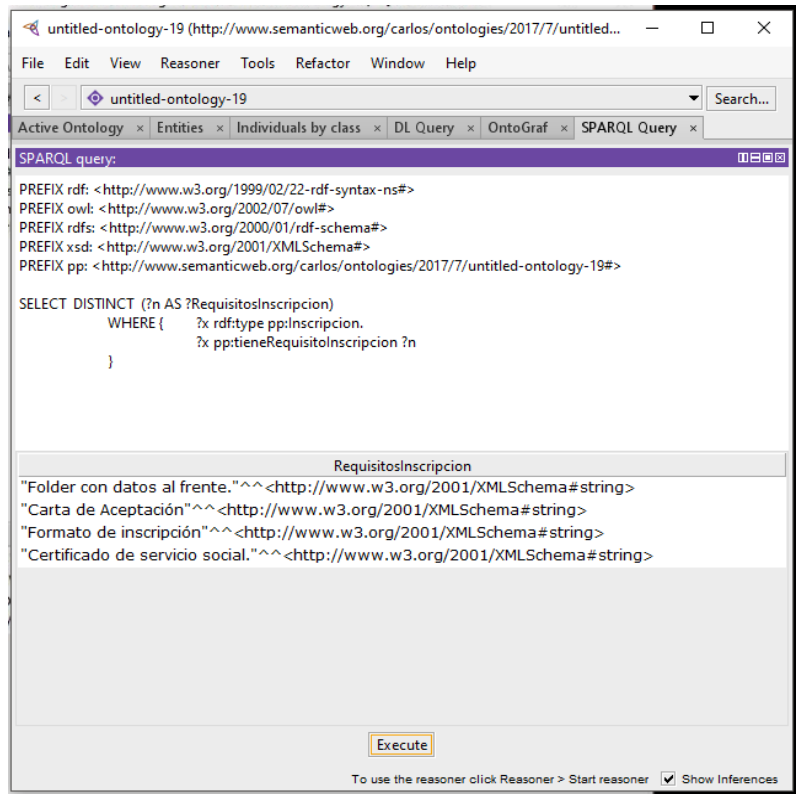

Fig. 5. Respuesta a la pregunta 3. 
Modelo ontológico para representar información sobre la práctica profesional ...

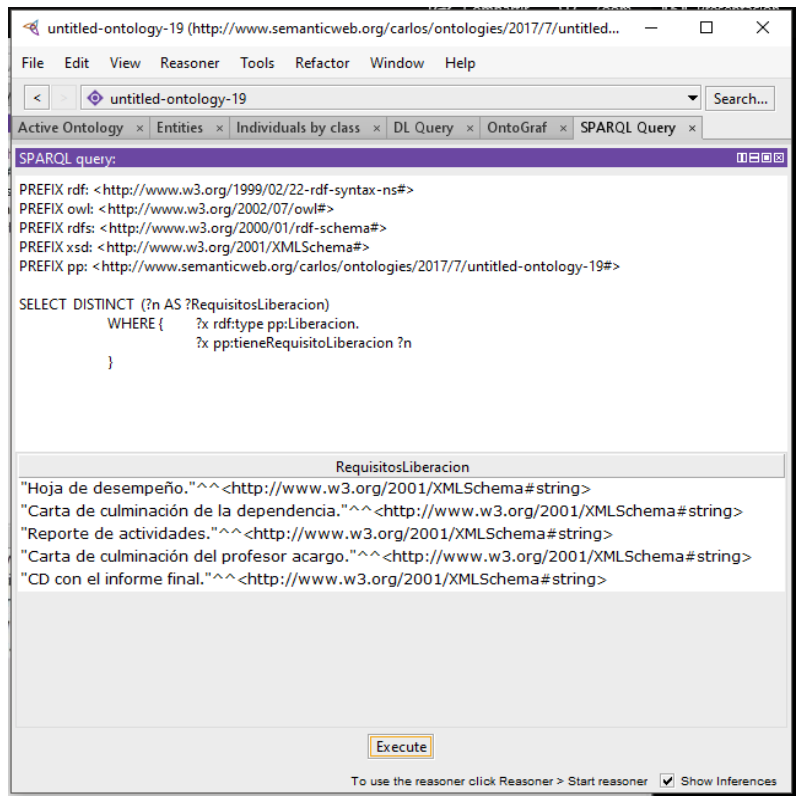

Fig. 6. Respuesta a la pregunta 4 .

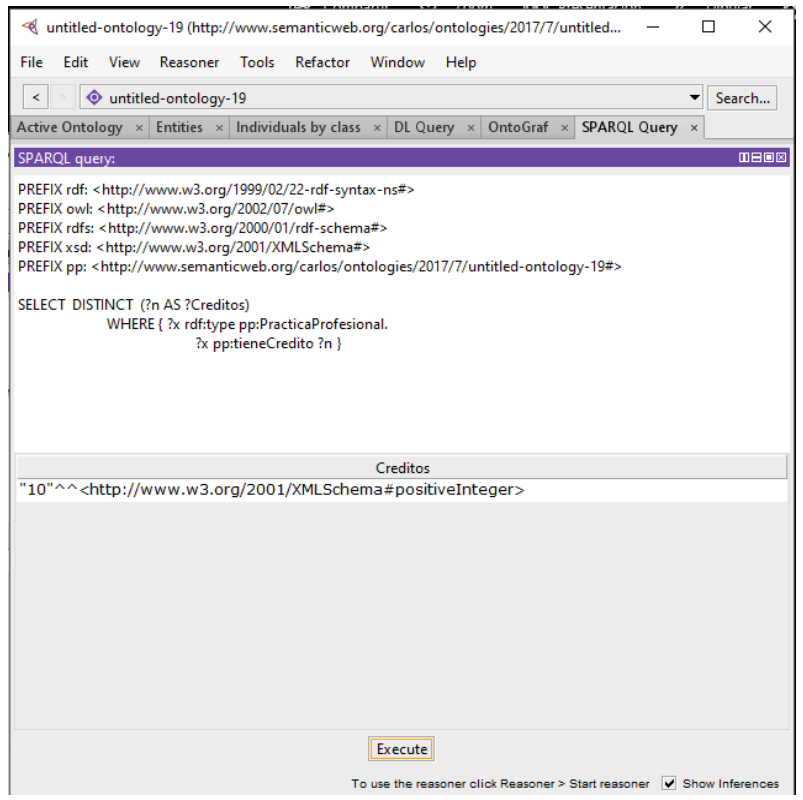

Fig. 7. Respuesta a la pregunta 5. 
Juan Carlos Flores Molina, Mireya Tovar Vidal, Ana Patricia Cervantes Márquez

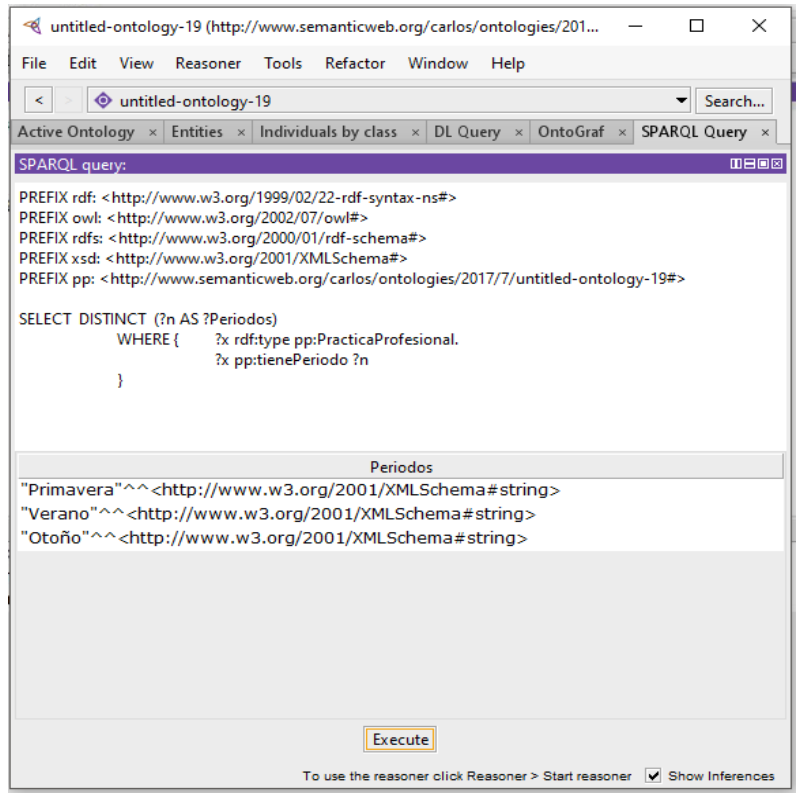

Fig. 8. Respuesta a la pregunta 6 .

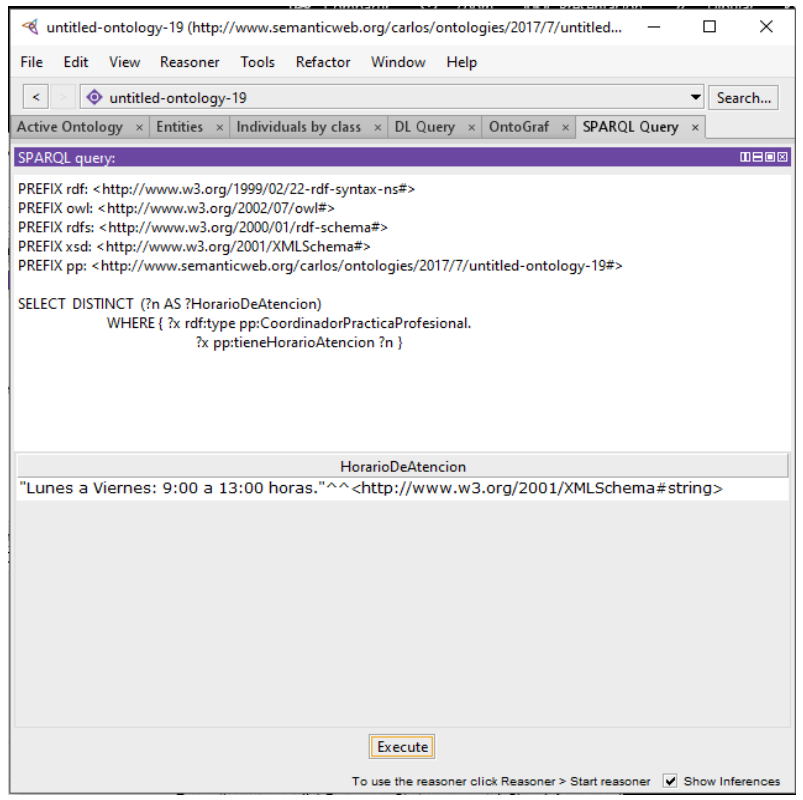

Fig. 9. Respuesta a la pregunta 7. 
Modelo ontológico para representar información sobre la práctica profesional ...

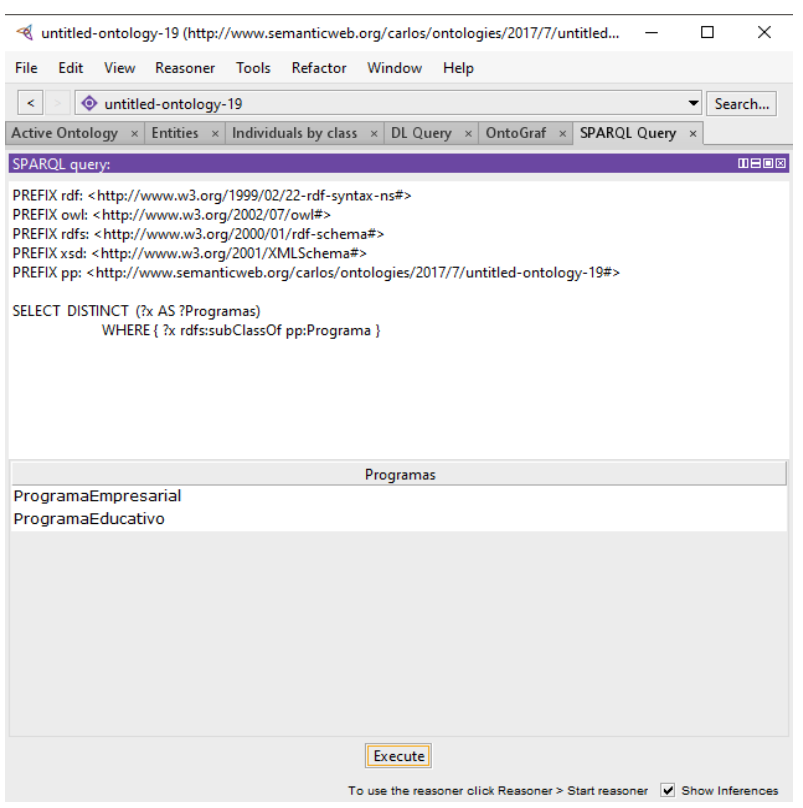

Fig. 10. Respuesta a la pregunta 8.

información acerca de trámites o servicios como es la práctica profesional a nivel superior. Como trabajo a futuro se pretende la creación de una aplicación que utilice la ontología para hacer el poblado autómatico a nivel de instancias de cada clase definida en la ontología y la presentación de las respuestas de las preguntas de competencia al usuario final.

Agradecimientos. Esta investigación es apoyada por el Fondo Sectorial de Investigación para la Educación, proyecto Conacyt CB/257357. Por el proyecto ID 00478 VIEP-BUAP y por el proyecto PRODEP-SEP ID 00570 (EXB-792) DSA/103.5/15/10854.

\section{Referencias}

1. Baez Bagatella, J.A., Tamborell Hérnandez, A., Lasserre Chávez, H., Ramos Flores, O., Tovar Vidal, M., Vilariño Ayala, D.: Un modelo ontológico para representar la organización de una unidad educativa. In: Avances recientes en ciencias computacionales - CICOMP 2016 (2016)

2. Bravo, M., Martínez Reyes, F., Rodríguez, J.: Representation of an academic and institutional context using ontologies. Reseach in Computing Science 87, 9-17 (2014)

3. DuCharme, B.: Learning SPARQL querying and updating with SPARQL 1.1. O'Reilly Media, Sebastopol, CA (2013) 
4. Gómez Pérez, A., Fernández López, M., Corcho, O.: Ontological Engineering with examples from the areas of knowledge Management e-Commerce and the Semantic Web. Springer (2004)

5. Gruber, T.R.: A translation approach to portable ontology specifications. Knowledge Acquisition 5(2), 199-220 (Jun 1993), http://dx.doi.org/10.1006/knac. 1993.1008

6. Mora Arciniega, M.B., Segarra Faggioni, V.: Modelo ontológico para la representación de datos académicos y su publicación con tecnología semántica. Opción 10, 267-282 (2016)

7. Musen, M.A.: The protégé project: A look back and a look forward. AI Matters 1(4), 4-12 (Jun 2015), http://doi.acm.org/10.1145/2757001.2757003

8. Reyes, C., Tovar, M., Vázquez, S.: Ontology for the description of a masters degree program in computer sciences. In: Proceedings of the XVIII International Conference on Human Computer Interaction. pp. 12:1-12:4. Interacción '17, ACM, New York, NY, USA (2017), http://doi.acm.org/10.1145/3123818.3123836

9. Rose Gómez, C.E., Serna Encias, M.T., Rodríguez Elías, O.M.: Uso del lenguaje natural para la búsqueda de respuestas en un sistema de gestión de conocimiento académico. Tecnologías emergentes y avances de la computación en México Primera Edición, 155-160 (2016)

10. Rosell León, Y., Senso Ruiz, J.A., Leiva Mederos, A.A.: Diseño de una ontología para la gestión de datos heterogéneos en universidades. Información en Ciencias de la Salud 27, 545-567 (2016)

11. Tabares García, J.J., Jiménez Builes, J.A.: Ontología para el proceso evaluativo en la educación superior. Revista Virtual Universidad Cat'olica del Norte 42, 68-79 (2014)

12. Tovar, M., Flores, J.C., Reyes, J.: An ontology for representing information over social service in an educational institution. In: Conference Special Session on Knowledge Discovery and Cloud Computing Applications. pp. 391-399 (01 2017) 